June 2019

\title{
Analysis of Candida auris fungemia at a single facility in Kenya
}

Rodney Adam

Aga Khan University, rodney.adam@aku.edu

Gunturu Revathi

Aga Khan University, gunturu.revathi@aku.edu

Nancy Okinda

Aga Khan University, nancy.okinda@aku.edu

Melanie Fontaine

Aga Khan University, melanie.fontaine@aku.edu

Jasmit Shah

Aga Khan University, jasmit.shah@aku.edu

See next page for additional authors

Follow this and additional works at: https://ecommons.aku.edu/eastafrica_fhs_mc_pathol Part of the Infectious Disease Commons, and the Pathology Commons

\section{Recommended Citation}

Adam, R., Revathi, G., Okinda, N., Fontaine, M., Shah, J., Kagotho, E., Castanheira, M., Pfaller, M. A., Maina, D. (2019). Analysis of Candida auris fungemia at a single facility in Kenya. International Journal of Infectious Diseases, 85, 182-187.

Available at: https://ecommons.aku.edu/eastafrica_fhs_mc_pathol/168 
Authors

Rodney Adam, Gunturu Revathi, Nancy Okinda, Melanie Fontaine, Jasmit Shah, Elizabeth Kagotho, Mariana Castanheira, Michael A. Pfaller, and Daniel Maina 


\title{
Analysis of Candida auris fungemia at a single facility in Kenya
}

\author{
Rodney D. Adam ${ }^{\mathrm{a}, *}$, Gunturu Revathi ${ }^{\mathrm{b}}$, Nancy Okinda ${ }^{\mathrm{b}}$, Melanie Fontaine ${ }^{\mathrm{b}}$, Jasmit Shah ${ }^{\mathrm{c} \text {, }}$, \\ Elizabeth Kagotho ${ }^{\mathrm{b}}$, Mariana Castanheira ${ }^{\mathrm{d}}$, Michael A. Pfaller ${ }^{\mathrm{d}}$, Daniel Maina ${ }^{\mathrm{b}}$ \\ a Departments of Pathology and Medicine, Aga Khan University, Third Parklands Rd., Nairobi, Kenya \\ ${ }^{\mathrm{b}}$ Department of Pathology, Aga Khan University, Third Parklands Rd., Nairobi, Kenya \\ ${ }^{\mathrm{c}}$ Departments of Population Health and Medicine, Aga Khan University, Third Parklands Rd., Nairobi, Kenya \\ d JMI Laboratories, Inc., North Liberty, IA 52317, USA
}

\section{A R T I C L E I N F O}

\section{Article history:}

Received 27 March 2019

Received in revised form 31 May 2019

Accepted 4 June 2019

Corresponding Editor: Eskild Petersen, Aarhus, Denmark

\section{Keywords:}

Candida auris

Candidemia

Carbapenems

Central venous catheters

Antimicrobial stewardship

\begin{abstract}
A B S T R A C T
Objectives: Candida auris emerged as a human pathogen in 2009 and has subsequently been identified around the world as a cause of invasive candidiasis. We did an analysis from a single institution in order to analyze risk factors and outcomes for $C$. auris candidemia.

Methods: Patients with candidemia were identified by the electronic medical record and reviewed for risk factors and outcome. Candida isolates were identified by Vitek2 as Candida haemulonii, but species determinations for 21 of the isolates using published molecular and proteomic methods identified all as C. auris.

Findings: From September 2010 to December 2016, C. auris accounted for 38\% of 201 patients with candidemia, while C. albicans contributed $25 \%$. C. auris patients had been hospitalized longer (mean 32 days vs. 13 days; $\mathrm{p}<0.001$ ), were more likely to have central lines preceding candidemia than $C$. albicans patients ( $84 \%$ vs. $54 \%$; $\mathrm{p}=<0.001$ ) and had more commonly been treated with carbapenems ( $83 \%$ vs $61 \%$ for C. albicans $[\mathrm{p}=0.01])$. The crude mortality was $29 \%$, compared to $36 \%$ for $C$. albicans.

Conclusions: These findings suggest an opportunistic pathogen that may be less virulent, but difficult to eradicate and that control efforts should focus on antimicrobial usage.

(c) 2019 The Authors. Published by Elsevier Ltd on behalf of International Society for Infectious Diseases. This is an open access article under the CC BY-NC-ND license (http://creativecommons.org/licenses/by-
\end{abstract} nc-nd/4.0/).

\section{Introduction}

Candidemia is currently the most commonly recognized form of invasive candidiasis and is most commonly seen in critical care settings where central vascular lines and broad-spectrum antibiotics are highly prevalent risk factors (Arendrup, 2010; Arendrup et al., 2011; Pfaller and Diekema, 2007; Pfaller et al., 2019). In series throughout the world, Candida species are the fourth leading cause of hospital-acquired bloodstream infection (BSI), accounting for 8 to $12 \%$ of all BSI acquired in United States hospitals (Wisplinghoff et al., 2004). In earlier series, Candida albicans was the dominant species, but in series reported since 2010 , it has formed only $50 \%$ of the isolates (Arendrup, 2010; Cleveland et al., 2015; Guinea, 2014). In comparison, Candida auris is a newly identified cause of invasive candidiasis and uncommon in most series. C. auris is part of a complex of related species that includes $C$. haemulonii, C. duobushaemulonii and C. lusitaniae and was recognized as a

\footnotetext{
* Corresponding author.

E-mail address: Rodney.adam@aku.edu (R.D. Adam).
}

human colonizer in 2009 (Satoh et al., 2009) and as a cause of invasive infection in 2011 (Lee et al., 2011). After its initial discovery in Asia, C. auris has been recognized as a relatively drugresistant yeast that can cause a wide spectrum of infections, ranging from fungemia to deep-seated infections, especially in intensive care settings (Chowdhary et al., 2014; Emara et al., 2015; Lee et al., 2011; Magobo et al., 2014; Sarma et al., 2013). Recent reports have suggested a unique susceptibility profile that includes highly elevated MICs to fluconazole, and reduced susceptibility to voriconazole, echinocandins, and flucytosine (Bidaud et al., 2018; Chowdhary et al., 2014; Chowdhary et al., 2018; Chowdhary et al., 2013; Hou et al., 2019; Lee et al., 2011; Magobo et al., 2014).

Candida species are the most common agents of hospitalacquired bloodstream infection at Aga Khan University Hospital Nairobi (AKUHN) (Maina et al., 2016) and C. auris has been the most commonly identified species of Candida since 2011. As such, the institution is ideally placed to provide information on risk factors and outcomes of invasive infections caused by this pathogen. The present study provides an evaluation of the risk factors and outcomes of candidemia due to $C$. auris in comparison with that caused by $C$. albicans and other Candida species. 


\section{Methods}

Patient information

AKUHN is a 280-bed referral center with 50 critical care beds. Patients treated at AKUHNN who had at least one blood culture between September 2010 and December 2016 that grew Candida species were included in the study and medical records were reviewed for patient details and comorbidities. The main objective of this study was to assess factors that may be influencing Candida species, duration and mortality.

The electronic medical record (EMR) was used to identify patients with candidemia and written records were reviewed to identify details not contained in the EMR. Positive blood cultures of the same species were considered to be part of the same episode if there was less than one month between successive positive blood cultures. For patients with more than one episode, the primary episode used for analysis was the species found in the first positive culture. In order to determine whether the candidemia was likely to be the cause of death, we performed a review of the medical record during the time surrounding the candidemia to look for clinical deterioration or death within the period of two days before to a week after the death and absence of a more likely cause for the deterioration.

The BD BACTEC ${ }^{\mathrm{TM}}$ (Franklin Lakes, New Jersey), USA was used for blood cultures and the VITEK ${ }^{\circledR} 2$ Compact (bioMérieux, Marcy-l'Étoile, France) for microbial identification and routine susceptibility testing. Vitek2 YST ID cards were used for yeast identification and Vitek2 AST YS07 for yeast susceptibility testing. The Clinical Laboratory Standards Institute (CLSI) M27A3 breakpoints were used for interpretation (Clinical and Laboratory Standards Institute. 2008. Reference method for broth dilution antifungal susceptibility testing of yeasts, 3 rd ed. Approved standard M27-A3. Clinical and Laboratory Standards Institute, Wayne, PA; Clinical and Laboratory Standards Institute
M27-S4. Reference Method for Broth Dilution Antifungal Susceptibility Testing of Yeasts: 4th Informational Supplement. Wayne, PA. CLSI, 2012).

Twenty-one of the isolates identified by Vitek2 as Candida haemulonii were subjected to molecular identification using ribosomal intervening transcribed sequence (ITS) sequencing and pulsed field gel electrophoresis (PFGE) typing as well as MALDI-TOF (Pfaller et al., 2012). All 21 were identified as C. auris, and by ITS sequence comparison had $85 \%$ identity with C. haemulonii (sensu stricto). Thus, these isolates are referred to herein as $C$. auris. These 21 isolates were susceptibility tested by reference broth microdilution according to the CLSI guidelines (CLSI, 2008).

\section{Ethics review}

The study protocol was approved by the Ethics Committee of AKUHN with a waiver of individual consent.

\section{Results}

Appearance of $\mathrm{C}$. auris

A total of 224 episodes of candidemia from September 2010 through December 2016 were seen in 201 patients (Table 1; Supplementary Table S1). The Vitek2 was first used for identification of yeasts in the laboratory in September 2010, but the first C. auris isolate was not seen until May 2011, suggesting the appearance of a new organism rather than recognition due to new identification method. $C$. auris rapidly became the most common Candida BSI isolate in 2012 followed by a general downward trend through 2016 (Figure 1). This occurred in the absence of any new or special infection control efforts or changes in antibacterial or antifungal usage. All 21 organisms evaluated by PFGE belonged to a single clade (data not shown).

Table 1

Patient characteristics, comorbidities and factors associated with Candida species.

\begin{tabular}{|c|c|c|c|c|c|c|}
\hline & & \multirow[t]{2}{*}{ C. auris $(\mathrm{n}=77)$} & \multirow[t]{2}{*}{ C. albicans $(\mathrm{n}=50)$} & \multirow[t]{2}{*}{ Other $(n=74)$} & \multicolumn{2}{|l|}{$\begin{array}{l}\text { Bivariate Analysis } \\
\text { P-value }\end{array}$} \\
\hline & & & & & C. auris - C. albicans & C. auris - other \\
\hline Age & Mean age, years (SD) & $58(20)$ & $48(30)$ & $56(25)$ & $\begin{array}{l}0.047 \\
\text { OR: } 1.02(1.00-1.03)\end{array}$ & 0.686 \\
\hline Sex & Male & $43(56 \%)$ & $26(52 \%)$ & $44(60 \%)$ & 0.718 & 0.742 \\
\hline \multicolumn{7}{|c|}{ Comorbid Risk Factors } \\
\hline & HIV & $4(5 \%)$ & $3(6.0 \%)$ & $6(8.1 \%)$ & 0.598 & 0.612 \\
\hline & Renal Failure before admission & $30(39 \%)$ & $12(24 \%)$ & $16(22 \%)$ & 0.087 & $\begin{array}{l}0.023 \\
\text { OR: } 2.31(1.13-4.75)\end{array}$ \\
\hline & Diabetes & $17(22 \%)$ & $12(24 \%)$ & $16(22 \%)$ & 0.632 & 0.831 \\
\hline & Hypertension & $17(22 \%)$ & $14(28 \%)$ & $21(28 \%)$ & 0.484 & 0.491 \\
\hline & Malignancy & $7(9 \%)$ & $8(16 \%)$ & $8(11 \%)$ & 0.326 & 0.738 \\
\hline \multicolumn{7}{|c|}{ Hospital Related Factors } \\
\hline & Renal Failure after admission & $12(25 \%)$ & $9(24 \%)$ & $15(26 \%)$ & 1.000 & 1.000 \\
\hline & Critical Care Unit & $61(79 \%)$ & $29(58 \%)$ & $54(73 \%)$ & $\begin{array}{l}0.016 \\
\text { OR: } 2.76(1.26-6.06)\end{array}$ & 0.446 \\
\hline & Mechanical Ventilation & $17(22 \%)$ & $10(20 \%)$ & $6(8 \%)$ & 0.895 & $\begin{array}{l}0.034 \\
\text { OR: } 3.17(1.17-8.57)\end{array}$ \\
\hline & Presence of CVC & $65(84 \%)$ & $27(54 \%)$ & $52(70 \%)$ & $\begin{array}{l}<0.001 \\
\text { OR: } 4.61(2.01-10.58)\end{array}$ & 0.051 \\
\hline & Mean days with CVC (SD) (of patients who had CVC $n=144$ ) & $12(3)$ & $11(5)$ & $10(5)$ & 0.437 & 0.068 \\
\hline & Mean days with CVC (SD) (all patients $n=201$ ) & $10(5)$ & $6(6)$ & $7(6)$ & $\begin{array}{l}0.001 \\
\text { OR: } 1.12(1.05-1.19)\end{array}$ & $\begin{array}{l}0.005 \\
\text { OR: } 1.08(1.02-1.15)\end{array}$ \\
\hline \multicolumn{7}{|c|}{ Admission to Diagnosis Duration } \\
\hline & Mean days (SD) & $32(26)$ & $13(12)$ & $17(27)$ & $\begin{array}{l}<0.001 \\
\text { OR: } 1.09(1.04-1.13)\end{array}$ & $\begin{array}{l}<0.001 \\
\text { OR: } 1.04(1.02-1.07)\end{array}$ \\
\hline Deat & $\mathrm{h}$ in hospital & $22(29 \%)$ & $18(36 \%)$ & $29(39 \%)$ & 0.493 & 0.183 \\
\hline
\end{tabular}

Categorical comparisons were performed using a 2-sided Fisher's exact test. Continuous comparisons were performed using Students t-test.

CVC, central venous catheter; HIV, human immunodeficiency virus. 


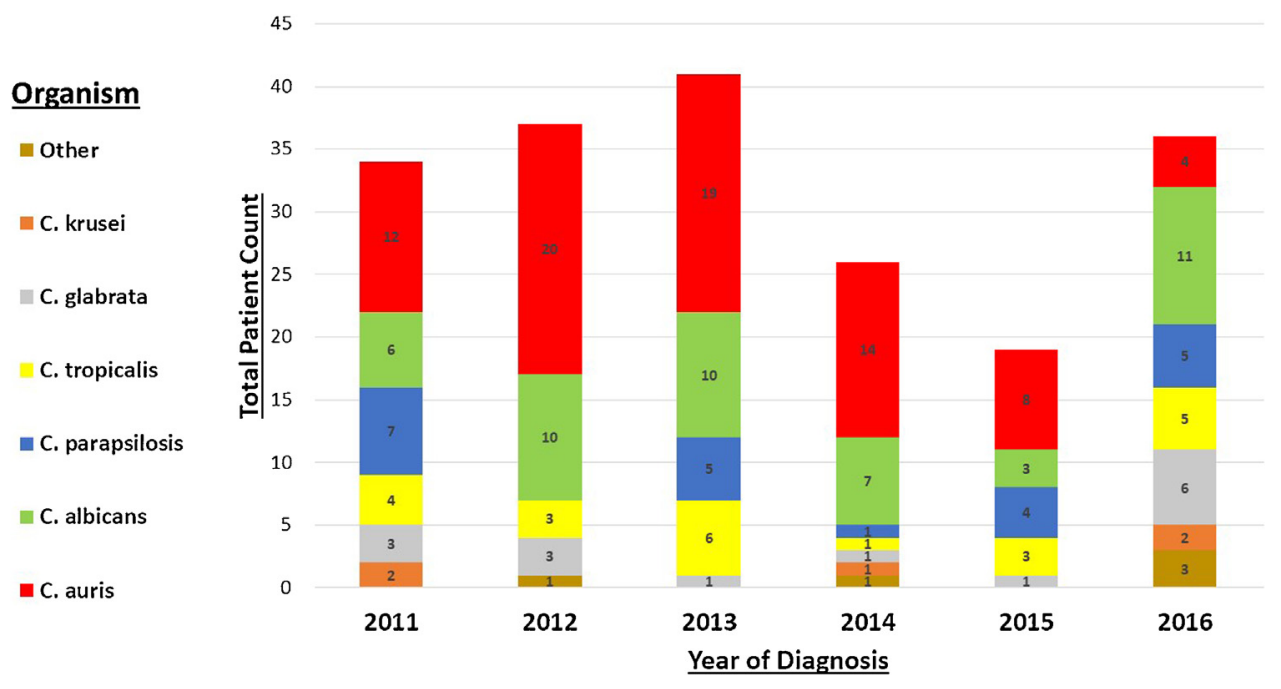

Figure 1. Yearly patterns for initial Candida infection from 2011 to 2016.

\section{General characteristics of patients with candidemia}

Of the 201 patients with candidemia, 131 (65\%) had at least one comorbidity identified. These did not differ significantly among the different species, although there was a trend toward more chronic kidney disease in $C$. auris patients (Table 1 ). The most common morbidities for all candidemia patients were chronic kidney disease, hypertension and diabetes: $7 \%$ were HIV-infected, reflecting the general patient population.

The majority of patients ( $72 \%$ ) were in a critical care unit at the time of their initial positive blood culture, with $16 \%$ requiring mechanical ventilation, $47 \%$ requiring dialysis and $72 \%$ requiring the placement of a central venous catheter (CVC). C. auris (79\%) and other non-albicans Candida patients (73\%) were more likely to be in a critical care unit than $C$. albicans patients $(58 \%$; $=0.016$ for the comparison of $C$. auris with $C$. albicans). C. auris patients were also more likely to have CVCs than $C$. albicans; a difference that persisted during a multivariate analysis (Table 3 ) with an odds ratio of $5.79(p=0.001)$. For the patients with CVCs, the number of days was also associated with $C$. auris in comparison with C. albicans as well as non-albicans Candida species (Table 1). There was also a borderline association of CVC with $C$. auris in comparison to other non-albicans species $(p=0.051)$, but this was not significant in the multivariate analysis.

\section{Antimicrobial treatment prior to candidemia}

Antibiotic use during the two week period prior to the candidemia was investigated to determine whether any antibiotics were more associated with $C$. auris than with other types of candidemia. During the two weeks prior to the initial blood culture that grew Candida, 89\% of patients had received an antibiotic. C. auris patients had more antibiotic days during the two weeks before their first positive blood culture and had been given a greater number of antibiotics during that time, in comparison with C. albicans and with other Candida species (Table 2). The antibiotic class that was the most highly associated with $C$. auris candidemia was the carbapenems (usually meropenem) (Table 2). C. auris

Table 2

Antimicrobial use during two weeks prior to diagnosis.

\begin{tabular}{|c|c|c|c|c|c|c|c|}
\hline & \multirow[t]{2}{*}{ C. auris $(\mathrm{n}=77)$} & \multirow[t]{2}{*}{ C. albicans $(\mathrm{n}=50)$} & \multirow[t]{2}{*}{ Others $(n=74)$} & \multicolumn{2}{|c|}{ C. auris - C. albicans Comparison } & \multicolumn{2}{|c|}{ C. auris - other Comparison } \\
\hline & & & & $\mathrm{OR}(\mathrm{CI})$ & P-value & $\mathrm{OR}(\mathrm{CI})$ & P-value \\
\hline \multicolumn{8}{|l|}{ Total antibacterial use, number (\%) } \\
\hline Antibacterial Use & $72(94 \%)$ & $44(88 \%)$ & $67(91 \%)$ & $1.96(0.57-6.82)$ & 0.288 & $1.50(0.46-4.97)$ & 0.503 \\
\hline Days of antibacterial, median (IQR) & $24(22)$ & $14(16)$ & $17(12)$ & $1.07(1.03-1.11)$ & $<0.001$ & $1.06(1.03-1.10)$ & $<0.001$ \\
\hline \multicolumn{8}{|l|}{ Number of Patient days for antibiotic class } \\
\hline Carbapenem & $8(4)$ & $6(4)$ & $7(4)$ & $1.16(1.03-1.30)$ & 0.014 & $1.09(1.00-1.20)$ & 0.062 \\
\hline Glyc & $8(6)$ & $6(5)$ & $6(5)$ & $1.08(0.96-1.23)$ & 0.223 & $1.10(1.01-1.22)$ & 0.046 \\
\hline Aminoglycoside & $6(4)$ & $4(5)$ & $5(4)$ & $1.12(0.94-1.39)$ & 0.235 & $1.08(0.91-1.30)$ & 0.415 \\
\hline Pen/BLI & $5(4)$ & $6(3)$ & $5(5)$ & $0.94(0.75-1.18)$ & 0.578 & $1.00(0.87-1.15)$ & 0.965 \\
\hline Linezolid & $6(4)$ & $2(1)$ & $5(4)$ & $1.74(1.13-3.76)$ & 0.062 & $1.09(0.90-1.33)$ & 0.384 \\
\hline \multicolumn{8}{|c|}{ Number of Patients Receiving Antibiotic Class, number (\%) } \\
\hline Carbapenem & $60(83)$ & $27(61)$ & $51(76)$ & $3.15(1.32-7.50)$ & 0.010 & $1.57(0.68-3.62)$ & 0.291 \\
\hline Glyc & $37(51)$ & $14(32)$ & $28(42)$ & $2.27(1.03-4.97)$ & 0.041 & $1.47(0.75-2.89)$ & 0.258 \\
\hline Aminoglycoside & $21(29)$ & $10(23)$ & $12(18)$ & $1.40(0.59-3.34)$ & 0.448 & $1.89(0.84-4.22)$ & 0.122 \\
\hline Pen/BLI & $26(36)$ & $9(21)$ & $22(33)$ & $2.20(0.92-5.28)$ & 0.078 & $1.16(0.57-2.33)$ & 0.685 \\
\hline Linezolid & $21(29)$ & $6(14)$ & $12(18)$ & $2.61(0.96-7.09)$ & 0.060 & $1.89(0.84-4.22)$ & 0.122 \\
\hline \multicolumn{8}{|l|}{ Antifungal Use Prior to Candidemia } \\
\hline Amphotericin & $4(5 \%)$ & $0(0 \%)$ & $0(0 \%)$ & - & - & - & - \\
\hline Echinocandin & $3(4 \%)$ & $1(2 \%)$ & $3(4 \%)$ & $0.9(0.08-11.2)$ & 0.959 & $2.0(0.32-12.24)$ & 0.453 \\
\hline Azole & $12(16 \%)$ & $5(10 \%)$ & $8(11 \%)$ & $0.3(0.03-3.56)$ & 0.370 & $1.6(0.31-7.87)$ & 0.593 \\
\hline
\end{tabular}

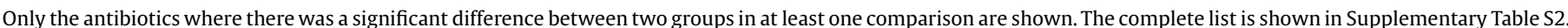

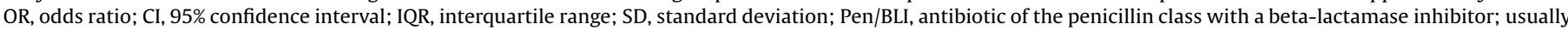
piperacillin/tazobactam, sometimes amoxicillin/clavulanate. 
Table 3

Multivariate analysis of $C$. auris vs. C. albicans.

\begin{tabular}{lll}
\hline Variable & OR (95\% CI) & P value \\
\hline Carbapenem (Days) & $1.19(1.05-1.35)$ & 0.007 \\
Carbapenem (Number) & $2.67(1.06-6.75)$ & 0.038 \\
Critical Care Unit & $3.14(1.02-9.72)$ & 0.047 \\
Presence of CVC & $5.79(2.12-15.80)$ & 0.001 \\
\hline
\end{tabular}

Based on the Univariate analysis: age, critical care unit and presence of CVC was significant when comparing $C$. auris and $C$. albicans. These variables were used as a forward selection in a logistic regression model. Four independent associations were found.

a) After adjusting for Critical care, with an increase in one unit of carbapenem days, the odds of being a C. auris candidemia are 1.19 .

b) After adjusting for carbapenem days, the odds of being a C. auris candidemia for those in Critical care unit over the odds of being a C. auris candidemia for those not in Critical care unit are 3.14.

c) After adjusting for presence of CVC, the odds of being a C. auris candidemia for those having had Carbapenem over the odds of being a $C$. auris candidemia for those not having Carbapenem are 2.67.

d) After adjusting for use of carbapenem, the odds of being a $C$. auris candidemia for those having a CVC over the odds of being a $C$. auris candidemia for those not having a CVC are 5.79 .

patients were more likely to have received carbapenems before the candidemia than C. albicans patients and had received more days of carbapenems than $C$. albicans or other candidemia patients. The independent association was confirmed in the multivariate analysis for $C$. auris vs. $C$. albicans, both for the frequency of usage and number of days (Table 3 ). There were weaker associations with the use of other antibiotics including penicillin/beta-lactamase combinations (primarily piperacillin/tazobactam) and linezolid. However, these associations were not confirmed as independent associations in the multivariate analyses. C. auris patients were more likely to have been treated with azoles than $C$. albicans patients (OR 3.2, p=0.052), although that included only $23 \%$ of C. auris patients (Supplementary Table S2).

In vitro susceptibilities of Candida isolates

Antifungal susceptibility testing was routinely performed for the Candida bloodstream isolates using the Vitek2 system and results are shown in Table 4 as an MIC range rather than susceptible vs. resistant. Although clinical breakpoints (CBPs) have not been established for $C$. auris, all isolates tested in the present study had fluconazole MICs of at least $16 \mathrm{mg} / \mathrm{L}$ and generally had voriconazole MICs at least two-fold higher than other Candida species. Caspofungin MIC values were generally one dilution higher than that of other species of Candida ( $\mathrm{MIC}_{90} 0.5 \mathrm{mg} / \mathrm{L}$ versus $<0.25 \mathrm{mg} / \mathrm{L}$, respectively) (Table 4). C. auris was routinely within the susceptible range for 5-flucytosine (data not shown), but this drug is not available in Kenya.

It should be noted that the Vitek2 system has not been shown to be reliable for testing C. auris (Kathuria et al., 2015). As such, the susceptibilities of 21 randomly selected $C$. auris isolates were also determined by the CLSI broth microdilution (BMD) method (see methods), which is generally considered to be the gold standard (Table 5). All isolates were susceptible to amphotericin (MIC, $\leq 1 \mathrm{mg} / \mathrm{L}$ ) and caspofungin (MIC, $\leq 0.25 \mathrm{mg} / \mathrm{L}$ ) and highly resistant (MIC $\geq 128 \mathrm{mg} / \mathrm{L}$ ) to fluconazole. There was no evidence of a paradoxical growth effect (Bidaud et al., 2018) with caspofungin. Similar to fluconazole, voriconazole MIC values were elevated with 17/21 (81\%) isolates tested by BMD showing MIC values at or above the resistant breakpoint of $\geq 1 \mathrm{mg} / \mathrm{L}$ established for C. albicans (CLSI, 2017).

There were $18 \mathrm{C}$. auris patients with documented candidemia of 14 days or longer, despite the lack of routine followup cultures in many patients, demonstrating the ability of this organism to cause prolonged candidemia. For these patients, we compared the MICs of the isolates to determine whether there was evidence for acquisition of in vitro resistance in these patients with prolonged candidemia. All Vitek2 susceptibility patterns of $C$. auris isolates from these patients are shown in Supplementary Table S4. There was frequently a difference in susceptibility of up to 4-fold, but no clear trend of increasing MIC with later isolates. Thus, there is not a clear pattern of increasing resistance over time for the agents tested.

\section{Outcome of patients with candidemia}

The mortality rate for $C$. auris patients trended toward being lower than seen for other Candida species, although not statistically significant (29\% vs. $36 \%$ for C. albicans and $39 \%$ for other Candida species). Since the $C$. auris patients tended to be older and had been hospitalized longer before the candidemia, we determined how often early mortality resulted and whether it was likely to be related to their candidemia. There was a longer duration from the initial positive blood culture for patients who died with $C$. auris than with C. albicans (Supplementary Figure S1). Of the 28 patients with candidemia who died within a week of their initial blood culture, records from 25 were available to review for the likely cause of death. We examined the records to determine the likelihood that the candidemia made a significant contribution to their deaths. The candidemia was a likely contributor to the deaths for only three patients (two C. albicans and one C. glabrata) (see Supplementary Table S5).

\section{Discussion}

C. auris emerged as a cause of candidemia in May 2011 and by the following year, had become the most common species. While it is possible that earlier cases had been present in our institution, it is notable that for the first eight months of using the current identification system, there were no cases suggestive of $C$. auris. In the absence of any specific interventions, the actual number and the percentage of cases subsequently decreased over time so that in 2016, there were five cases comprising $14 \%$ of the total candidemia cases, down from a peak of 59\% in 2012.

Risk factors for candidemia that have been identified in nonneutropenic hospitalized patients include critical care treatment,

Table 4

Candida susceptibilities determined by Vitek2.

\begin{tabular}{|c|c|c|c|c|c|c|c|c|c|c|c|c|c|}
\hline \multirow[b]{2}{*}{$\begin{array}{l}\text { MIC } \\
(\mathrm{mg} / \mathrm{L})\end{array}$} & \multicolumn{4}{|c|}{ Caspofungin } & \multicolumn{5}{|c|}{ Fluconazole } & \multicolumn{4}{|c|}{ Voriconazole } \\
\hline & $\mathrm{n}$ & $\leq 0.25$ & 0.5 & 1 & $\mathrm{n}$ & $\leq 1$ & $2-8$ & $16-32$ & $\geq 64$ & $\mathrm{n}$ & $\leq 0.5$ & 1 & $\geq 2$ \\
\hline C. auris $(n=72)$ & 34 & 20 & 14 & 0 & 72 & 0 & 0 & 18 & 54 & 72 & 10 & 47 & 5 \\
\hline C. albicans $(n=41)$ & 23 & 22 & 1 & 1 & 41 & 37 & 21 & 0 & 2 & 41 & 38 & 2 & 1 \\
\hline C. parapsilosis $(n=14)$ & 10 & 1 & 3 & 6 & 14 & 7 & 1 & 1 & 5 & 14 & 9 & 2 & 3 \\
\hline C. tropicalis $(n=17)$ & 11 & 11 & 0 & 0 & 17 & 16 & 1 & 0 & 0 & 17 & 17 & 0 & 0 \\
\hline C. glabrata $(n=15)$ & 10 & 10 & 0 & 0 & 15 & 0 & 14 & 0 & 1 & 15 & 14 & 1 & 3 \\
\hline
\end{tabular}

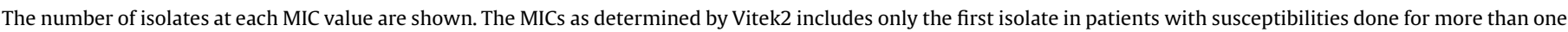
isolate. The number of isolates at each MIC range are noted without inferred susceptibility or resistance. 
Table 5

C. auris susceptibilities determined by broth microdilution (mg/L).

\begin{tabular}{|c|c|c|c|c|c|c|c|c|c|c|}
\hline \multirow[b]{2}{*}{$\begin{array}{l}\text { MIC } \\
(\mathrm{mg} / \mathrm{L})\end{array}$} & \multirow{2}{*}{$\begin{array}{l}\text { Amphotericin } \\
1\end{array}$} & \multicolumn{4}{|c|}{ Caspofungin } & \multicolumn{2}{|c|}{ Fluconazole } & \multicolumn{3}{|c|}{ Voriconazole } \\
\hline & & 0.03 & 0.06 & 0.12 & 0.25 & 128 & $>128$ & 0.5 & 1 & 2 \\
\hline C. auris $(\mathrm{n}=21)$ & 21 & 1 & 1 & 6 & 8 & 6 & 15 & 4 & 10 & 7 \\
\hline
\end{tabular}

diabetes, renal failure, vascular lines and broad spectrum antibiotics (Adams et al., 2018; Chow et al., 2018; Lockhart et al., 2017). Thus, it is notable in the current study, some of these risk factors were much more common in $C$. auris patients than in C. albicans patients. Notably, a CVC was present (fulfilling NHSN criteria for CLABSI) in $84 \%$ of $C$. auris patients and only $54 \%$ of C. albicans patients. The use of broad spectrum antibiotics was also notable, since broad spectrum antibiotics, particularly carbapenems, have been documented as risk factors for $C$. albicans candidemia. In the current series, carbapenems were used more frequently ( $83 \% \mathrm{vs.}$ $61 \%$ ) and for more days ( 8 vs. 6 ) for C. auris than for C. albicans patients.

The largest study of the clinical characteristics of patients with $C$. auris fungemia included 74 patients with $C$. auris candidemia from 19 ICUs in India, representing 5.3\% of the Candida isolates (Rudramurthy et al., 2017). They found that C. auris was associated with longer ICU stay, pre-existing respiratory illness, antifungal exposure and low APACHE II scores. They did not find an association with presence of a CVC, but $C$. auris was associated with longer duration of central venous catheterization. Exposure to antibacterial agents was not reported in the India-based study.

The 30-day mortality of $41.9 \%$ and an estimated $27 \%$ attributable mortality was higher than that of other Candida species (Rudramurthy et al., 2017). In comparison, a study that included 41 C. auris patients from multiple continents with clinical outcome data reported a crude mortality rate of 59\% (Lockhart et al., 2017). In contrast, the in-hospital mortality in the current series was $29 \%$, which was actually lower than for other Candida species, even though they were older and had been hospitalized longer. A review of the records for patients who died within one week of their initial documented candidemia revealed little evidence for a substantial contribution of $C$. auris to their mortality.

The antifungal susceptibilities for $C$. auris isolates in the current series were more resistant to antifungal agents than other species already known to have reduced susceptibility to antifungal agents. For example, $93 \%$ of C. glabrata isolates had a voriconazole MIC $\leq 0.5 \mathrm{mg} / \mathrm{L}$, but only $14 \%$ of $C$. auris had that level of susceptibility when tested by the Vitek2 system. The $C$. auris isolates were susceptible to echinocandins (caspofungin) by Vitek2 testing, but the MICs were higher than for other isolates. For example, $42 \%$ of $C$. auris isolates had caspofungin MICs $\geq 0.5 \mathrm{mg} / \mathrm{L}$, a level that was rare for species other than C. parapsilosis. In contrast to the Vitek2 results, the microdilution MICs for caspofungin were lower, MICs 0.12 or $0.25 \mathrm{mg} / \mathrm{L}$ for most isolates. All 21 isolates that were tested by microdilution for amphotericin had MICs of $1 \mathrm{mg} / \mathrm{L}$, which is within the susceptible range. It must be recognized that Vitek2 results for $C$. auris have been reported to be less reliable when compared to that of BMD or Etest (Kathuria et al., 2015) and this should be considered to be a limitation of this data.

These results are similar to the susceptibility patterns for C. auris from the Indian ICU study (Rudramurthy et al., 2017), in which the MIC50 for amphotericin was $1 \mathrm{mg} / \mathrm{L}$ and most voriconazole MICs were 0.5 or $1 \mathrm{mg} / \mathrm{L}$. However, most of their caspofungin MICs ranged from 0.25 to $2 \mathrm{mg} / \mathrm{L}$; similar to our Vitek2 results but higher than our BMD results. A study of 51 isolates from New York also demonstrated similar MICs with voriconazole ranging from 0.5 to $4 \mathrm{mg} / \mathrm{L}$ ( $\mathrm{MIC}_{50} 2 \mathrm{mg} / \mathrm{L}$ ), caspofungin 0.03 to $0.25 \mathrm{mg} / \mathrm{L}\left(\mathrm{MIC}_{50} 0.06 \mathrm{mg} / \mathrm{L}\right.$ ), and amphotericin 0.5 to $4 \mathrm{mg} / \mathrm{L}\left(\mathrm{MIC}_{50} 1.5 \mathrm{mg} / \mathrm{L}\right)$. All series have shown a high level of resistance to fluconazole. A study that included $54 \mathrm{C}$. auris isolates and included 25 patients with candidemia also demonstrated similar susceptibility patterns with voriconazole, caspofungin and amphotericin $\mathrm{MIC}_{50} \mathrm{~S}$ of 2, 0.25 and $1 \mathrm{mg} / \mathrm{L}$, respectively (Lockhart et al., 2017).

The prolonged candidemia in a significant portion of patients despite the removal of CVCs in most cases as well as treatment with agents that have in vitro activity raises the question of how the organism persists in the intravascular space of the patient. This discrepancy between in vitro activity and clinical nonresponse suggests that biologic reasons for the lack of treatment response should be explored, considering host and organism factors. One hypothesis is that the persistence is due to intravascular infection (e.g. central vein suppurative phlebitis), perhaps through biofilm production. A recent study has demonstrated the production of biofilm by $C$. auris, although less robust than seen for $C$. albicans (Larkin et al., 2017). Further clinical data are required to determine the best treatment approaches for $C$. auris, especially in view of the prolonged candidemia that is frequently seen despite CVC removal and treatment with agents that are active in vitro.

In summary, this is an organism with a course that is typically indolent, but has the ability to cause prolonged fungemia and is of particular concern because of its reduced susceptibility to antifungal agents. It is likely that the most effective preventive measure will consist of reduced exposure of patients to antibiotics, particularly the carbapenems.

\section{Conflict of interest}

This research did not receive any specific grant from funding agencies in the public, commercial, or not-for-profit sectors.

None of the authors has a conflict of interest to report.

\section{Roles of the authors}

Rodney D. Adam: Coordinated project; data collection and analysis, writing.

Gunturu Revathi: Supervised microbiology work; data analysis, writing.

Nancy Okinda: Data collection and analysis.

Melanie Fontaine: Data collection and analysis.

Jasmit Shah: Data analysis.

Elizabeth Kagotho: Data collection and analysis.

Mariana Castanheira: Candida identification and susceptibility testing.

Michael A. Pfaller: Candida identification and susceptibility testing, writing.

Daniel Maina: Data collection and analysis.

\section{Acknowledgments}

We appreciate the work of the AKU microbiology laboratory for their role in making this possible. 
These data were presented in part at IDWeek 2018 and in a more preliminary form at ECCMID 2014.

\section{Appendix A. Supplementary data}

Supplementary material related to this article can be found, in the online version, at doi:https://doi.org/10.1016/j.ijid.2019.06.001.

\section{References}

Adams E, Quinn M, Tsay S, Poirot E, Chaturvedi S, Southwick K, et al. Candida auris in healthcare facilities, New York, USA, 2013-2017. Emerg Infect Dis 2018;24 (10):1816-24.

Arendrup MC. Epidemiology of invasive candidiasis. Curr Opin Crit Care 2010;16 (5):445-52.

Arendrup MC, Sulim S, Holm A, Nielsen L, Nielsen SD, Knudsen JD, et al. Diagnostic issues, clinical characteristics, and outcomes for patients with fungemia. J Clin Microbiol 2011;49(9):3300-8.

Bidaud AL, Chowdhary A, Dannaoui E. Candida auris: an emerging drug resistant yeast - a mini-review. J Mycol Med 2018;28(3):568-73.

Chow NA, Gade L, Tsay SV, Forsberg K, Greenko JA, Southwick KL, et al. Multiple introductions and subsequent transmission of multidrug-resistant Candida auris in the USA: a molecular epidemiological survey. Lancet Infect Dis 2018;18 (12):1377-84.

Chowdhary A, Anil Kumar V, Sharma C, Prakash A, Agarwal K, Babu R, et al. Multidrug-resistant endemic clonal strain of Candida auris in India. Eur J Clin Microbiol Infect Dis 2014;33(6):919-26.

Chowdhary A, Prakash A, Sharma C, Kordalewska M, Kumar A, Sarma S, et al. A multicentre study of antifungal susceptibility patterns among 350 Candida auris isolates (2009-17) in India: role of the ERG11 and FKS1 genes in azole and echinocandin resistance. J Antimicrob Chemother 2018;73(4):891-9.

Chowdhary A, Sharma C, Duggal S, Agarwal K, Prakash A, Singh PK, et al. New clonal strain of Candida auris, Delhi, India. Emerg Infect Dis 2013;19(10):1670-3.

Cleveland AA, Harrison LH, Farley MM, Hollick R, Stein B, Chiller TM, et al. Declining incidence of candidemia and the shifting epidemiology of Candida resistance in two US metropolitan areas, 2008-2013: results from population-based surveillance. PLoS One 2015;10(3)e0120452.

CLSI. Clinical and Laboratory Standards Institute M27-S4. Reference method for broth dilution antifungal susceptibility testing of yeasts. Third ed. Wayne, PA, USA: Clinical and Laboratory Standards Institute; 2008.

CLSI. M27 - Reference method for broth dilution antifungal susceptibility testing of yeasts. Fourth ed. Wayne, PA, USA: Clinical and Laboratory Standards Institutes; 2017.

Emara M, Ahmad S, Khan Z, Joseph L, Al-Obaid I, Purohit P, et al. Candida auris candidemia in Kuwait, 2014. Emerg Infect Dis 2015;21(6):1091-2.
Guinea J. Global trends in the distribution of Candida species causing candidemia. Clin Microbiol Infect 2014;20:5-10.

Hou X, Lee A, Jiménez-Ortigosa C, Kordalewska M, Perlin DS, Zhao Y. Rapid detection of ERG11-associated azole resistance and FKS-associated echinocandin resistance in Candida auris. Antimicrob Agents Chemother 2019;63(1) e01811-18.

Kathuria S, Singh PK, Sharma C, Prakash A, Masih A, Kumar A, et al. Multidrugresistant Candida auris misidentified as Candida haemulonii: characterization by matrix-assisted laser desorption ionization-time of flight mass spectrometry and DNA sequencing and its antifungal susceptibility profile variability by Vitek 2, CLSI broth microdilution, and Etest method. J Clin Microbiol 2015;53 (6): $1823-30$.

Larkin E, Hager C, Chandra J, Mukherjee PK, Retuerto M, Salem I, et al. The emerging pathogen Candida auris: growth phenotype, virulence factors, activity of antifungals, and effect of SCY-078, a novel glucan synthesis inhibitor, on growth morphology and biofilm formation. Antimicrob Agents Chemother 2017;61(5).

Lee WG, Shin JH, Uh Y, Kang MG, Kim SH, Park KH, et al. First three reported cases of nosocomial fungemia caused by Candida auris. J Clin Microbiol 2011;49 (9):3139-42.

Lockhart SR, Etienne KA, Vallabhaneni S, Farooqi J, Chowdhary A, Govender NP, et al. Simultaneous emergence of multidrug-resistant Candida auris on 3 continents confirmed by whole-genome sequencing and epidemiological analyses. Clin Infect Dis 2017;64(2):134-40.

Magobo RE, Corcoran C, Seetharam S, Govender NP. Candida auris-associated candidemia, South Africa. Emerg Infect Dis 2014;20(7):1250-1.

Maina D, Omuse G, Revathi G, Adam RD. Spectrum of microbial diseases and resistance patterns at a private teaching hospital in Kenya: implications for clinical practice. PLoS One 2016;11(1)e0147659.

Pfaller MA, Diekema DJ. Epidemiology of invasive candidiasis: a persistent public health problem. Clin Microbiol Rev 2007;20(1):133-63.

Pfaller MA, Diekema DJ, Turnidge JD, Castanheira M, Jones RN. Twenty years of the SENTRY antifungal surveillance program: results for Candida species from 1997-2016. Open Forum Infect Dis 2019;6(Supplement_1):S79-94.

Pfaller MA, Woosley LN, Messer SA, Jones RN, Castanheira M. Significance of molecular identification and antifungal susceptibility of clinically significant yeasts and moulds in a global antifungal surveillance programme. Mycopathologia 2012;174(4):259-71.

Rudramurthy SM, Chakrabarti A, Paul RA, Sood P, Kaur H, Capoor MR, et al. Candida auris candidaemia in Indian ICUs: analysis of risk factors. J Antimicrob Chemother 2017;72(6):1794-801.

Sarma S, Kumar N, Sharma S, Govil D, Ali T, Mehta Y, et al. Candidemia caused by amphotericin B and fluconazole resistant Candida auris. Indian J 2013;31(1):90-1.

Satoh K, Makimura K, Hasumi Y, Nishiyama Y, Uchida K, Yamaguchi H. Candida auris sp. nov., a novel ascomycetous yeast isolated from the external ear canal of an inpatient in a Japanese hospital. Microbiol Immunol 2009;53(1):41-4.

Wisplinghoff H, Bischoff T, Tallent SM, Seifert H, Wenzel RP, Edmond MB. Nosocomial bloodstream infections in US hospitals: analysis of 24,179 cases from a prospective nationwide surveillance study. Clin Infect Dis 2004;39 (3):309-17. 